\title{
Quivers of Bound Path Algebras and Bound Path Coalgebras
}

\author{
${ }^{1}$ Intan Muchtadi-Alamsyah \& ${ }^{2}$ Hanni Garminia \\ ${ }^{1}$ Algebra Research Division, FMIPA ITB, Bandung, Indonesia. \\ e-mail: ntan@math.itb.ac.id \\ ${ }^{2}$ Algebra Research Division, FMIPA ITB, Bandung, Indonesia. \\ e-mail: hani@math.itb.ac.id
}

\begin{abstract}
Algebras and coalgebras can be represented as quiver (directed graph), and from quiver we can construct algebras and coalgebras called path algebras and path coalgebras. In this paper we show that the quiver of a bound path coalgebra (resp. algebra) is the dual quiver of its bound path algebra (resp. coalgebra).
\end{abstract}

Keywords: quiver; bound path algebras; bound path coalgebras.

\section{$1 \quad$ Introduction}

Algebras can be represented as quiver (directed graph) and modules can be represented as quiver representation [1]. In [2] it is explained how to construct algebras from quivers called path algebras. In coalgebra representation theory, Chin [3] explained how to represent coalgebras as quiver, and also how to construct coalgebras form quivers called path coalgebras.

A finite dimensional algebra $A$ over an algebraically closed field $K$ is called basic if the quotient algebra of $A$ modulo the Jacobson radical is isomorphic to a product of $K$ as $K$-algebras. A theorem due to Gabriel says that a basic $K$ algebra is isomorphic to the factor algebra of the path algebra $K Q_{\mathrm{A}}$ by an admissible ideal, where $Q_{\mathrm{A}}$ is the quiver of A (see [1], [4]). Since any finite dimensional algebra is Morita equivalent to a uniquely determined basic algebra, it follows that any finite dimensional algebra A over an algebraically closed field is Morita equivalent to $K Q_{\mathrm{A}}$ modulo an admissible ideal.

Dually, a finite dimensional coalgebra $C$ is called pointed if each simple sub 7 coalgebra is of dimension one. As a dual of a result due to Gabriel, Chin and Montgomery [5] proved that any pointed coalgebra is isomorphic to a large subcoal-gebra of the path coalgebra of the quiver $C$. Since any coalgebra is Morita-Takeuchi equivalent to a uniquely determined pointed coalgebra, it follows that any coalgebra $C$ over an algebraically closed field is Morita-

Received January $19^{\text {th }}, 2010$, Revised May $31^{\text {st }}, 2010$, Accepted for publication June $7^{\text {th }}, 2010$. 
Takeuchi equivalent to a large subcoalgebra of the path coalgebra of quiver of C.

In [6] it is shown that the quiver of a path algebra (resp. path coalgebra) is the dual quiver of its path coalgebra (resp. algebra). In this paper we show that the same results hold for bound path algebras and bound path coalgebras. We show that the quiver of a bound path algebra (resp. bound path coalgebra) is the dual quiver of its bound path coalgebra (resp. bound path algebra), hence the dual of basic algebras are pointed coalgebras, and vice versa.

The paper is organized as follows: in section 2 we will give definition of path algebras and path coalgebras. In section 3 we will explain about dual path algebras and path coalgebras. Finally in the last section we will explain the main theorem about obtaining quivers of bound path algebras from quivers of bound path coalgebras and vice versa.

\section{$2 \quad$ Path Algebras and Path Coalgebras}

A quiver $Q$ is a quadruple $\left(Q_{0}, Q_{l}, s, t\right)$ where $Q_{0}$ is the set of vertices (points), $Q_{1}$ is the set of arrows and for each arrow $\alpha \in Q_{1}$, the vertices $s(\alpha)$ and $t(\alpha)$ are the source and the target of $\alpha$, respectively, see [1]. If $i$ and $j$ are vertices, an (oriented) path in $Q$ of length $m$ from $i$ to $j$ is a formal composition of arrows.

$$
\mathrm{p}=\alpha_{1} \alpha_{2} \ldots \alpha_{\mathrm{m}}
$$

where $s\left(\alpha_{1}\right)=i, t\left(\alpha_{m}\right)=j$ and $t\left(\alpha_{k-1}\right)=s\left(\alpha_{k}\right)$, for $k=2, \ldots, m$. To any vertex $i \in Q_{0}$ we attach a trivial path of length 0 , say $e_{i}$, starting and ending at $i$ such that for any arrow $\alpha$ (resp. $\beta$ ) such that $s(\alpha)=i$ (resp. $t(\beta)=$ $i)$ then $e_{i} \alpha=\alpha$ (resp. $\left.\beta e_{i}=\beta\right)$. We identify the set of vertices and the set of trivial paths.

Let $K Q$ be the $K$-vector space generated by the set of all paths in $Q$. Then $K Q$ can be endowed with a structure of $K$-algebra with multiplication induced by concatenation of paths, that is,

$$
\left(\beta_{1} \beta_{2} \ldots \beta_{m}\right)\left(\alpha_{1} \alpha_{2} \ldots \alpha_{n}\right)=\left\{\begin{array}{c}
\beta_{1} \beta_{2} \ldots \beta_{m} \alpha_{1} \alpha_{2} \ldots \alpha_{n} \text { if } t\left(\beta_{m}\right)=s\left(\alpha_{1}\right) \\
0, \text { otherwise }
\end{array}\right.
$$

$K Q$ is called the path algebra of the quiver $Q$. The algebra $K Q$ can be graded by 


$$
\mathrm{K} \mathrm{Q}=\mathrm{K} \mathrm{Q}_{0} \oplus \mathrm{KQ}_{1} \oplus \ldots \oplus \mathrm{K} \mathrm{Q}_{\mathrm{m}} \oplus \ldots,
$$

where $Q_{m}$ is the set of all paths of length $m$.

Definition 1 Let $Q$ be a finite connected quiver. The ideal of path algebra $K Q$ generated by arrows of $Q$ is called arrow ideal and denoted by $R_{Q}$.

Definition 2 Let $Q$ be a finite quiver and $R_{Q}$ be the arrow ideal in path algebra $K Q$. An ideal $I$ in $K Q$ is admissible if there exists $m \geq 2$ such that

$$
\mathrm{R}^{\mathrm{m}} \mathrm{Q} \subseteq \mathrm{I} \subseteq \mathrm{R}^{2} \mathrm{Q}
$$

If $I$ is an admissible ideal in $K Q,(Q, I)$ is called bound quiver. The quotient algebra $K Q / I$ is called bound path algebra.

The path algebra $K Q$ can be viewed as a graded $K$-coalgebra with comultiplication induced by the decomposition of paths, that is, if $p=\alpha_{1} \alpha_{2} \ldots \alpha_{m}$ is a path from the vertex $i$ to the vertex $j$, then

$$
\Delta(p)=e_{i} \otimes p+p \otimes e_{j}+\sum_{i=1}^{m-1} \alpha_{1} \ldots \alpha_{i} \otimes \alpha_{i+1} \ldots \alpha_{m}=\sum_{\tau v=p} \tau \otimes v
$$

and for a trivial path $e_{i}$ we have $\Delta(e i)=e i \otimes e i$. The counit of $K Q$ is defined by the formula

$$
\varepsilon(\alpha)=\left\{\begin{array}{c}
1, \text { if } \alpha \in Q_{0} \\
0, \text { if } \alpha \text { is a pathof length } \geq 1
\end{array}\right.
$$

The coalgebra $(K Q, \Delta, \varepsilon)$ is the path coalgebra of the quiver $Q$. For the convenience we denote by $K Q$ the path algebra of $Q$ and by $C Q$ the path coalgebra of $Q$.

Definition 3 A subcoalgebra of a path coalgebra is said to be admissible if it contains the subcoalgebra generated by all vertices and all arrows, that is, $C Q_{0}$ $\oplus C Q_{1}$ (see [7]). A subcoalgebra $C$ of a path coalgebra $C Q$ is called a relation subcoalgebra (see [8]) if $C$ is admissible and $C=\oplus_{a, b \in Q 0} C \cap C Q(a, b)$ where $C Q(a, b)$ is the subspace generated by all paths starting at $a$ and ending at $b$. 


\section{Dual Path Algebras and Path Coalgebras}

\subsection{Dual Path Coalgebras}

Let $Q$ be a quiver and $C Q$ and $K Q$ be the corresponding path coalgebra and path algebra, respectively. A basis of $K$-vector space $D(C Q)(D(K Q))$ consists of $p^{*}$ for all path $p, q \in Q$, where $p^{*}(q)=\delta_{p q}$, with $\delta$ is the delta Kronecker. Denote by $e_{1}, \cdots, e_{n}$ the trivial paths in $Q$. We define the multiplication map $\mu: D(C Q) \otimes D(C Q) \rightarrow D(C Q)$ and the unit map $\eta: K \rightarrow D(C Q)$ as following, for all $p^{*}, q^{*}$ basis $D(C Q)$,

$$
\begin{aligned}
& \mu\left(p^{*} \otimes q^{*}\right)=\left\{\begin{array}{r}
(q p) * \text { if } t(q)=s(p) \\
0 \text { otherwise }
\end{array}\right. \\
& \eta(1)=\mathrm{e}_{1}^{*}+\mathrm{e}_{2}^{*}+\cdots+\mathrm{e}_{\mathrm{n}}^{*} .
\end{aligned}
$$

Lemma 4 The multiplication $\mu$ and the unit $\eta$ satisfy

1. $\mu(i d \otimes \mu)=\mu(\mu \otimes i d)$

2. $(i d \otimes \mu)(\eta \otimes i d)=(\eta \otimes i d)(i d \otimes \mu)$

\section{Proof}

1. It is enough to show for basis elements of $D(C Q)$. Let $p^{*}, q^{*}$ and $r^{*}$ in basis of $D(C Q)$ where rqp is a path. Then

$$
\begin{aligned}
& \mu(i d \otimes \mu)\left(p^{*} \otimes q^{*} \otimes r^{*}\right)=\mu\left(p^{*} \otimes(r q)^{*}\right)=(r q p)^{*} \\
& \mu(\mu \otimes i d)\left(p^{*} \otimes q^{*} \otimes r^{*}\right)=\mu\left((q p)^{*} \otimes r^{*}\right)=(r q p)^{*}
\end{aligned}
$$

2. Let $q^{*}$ and $r^{*}$ in basis of $D(C Q)$ where $r q$ is a path.

$((i d \otimes \mu) \circ(\eta \otimes i d))(1 \otimes q * \otimes r *)=(i d \otimes \mu)\left(\left(\sum_{i=1}^{n} e_{i} *\right) \otimes q * \otimes r *\right)=\left(\sum_{i=1}^{n} e_{i}^{*}\right) \otimes(r q) *=(r q) *$
$((\eta \otimes i d) \circ(i d \otimes \mu))(1 \otimes q * \otimes r *)=(\eta \otimes i d)(1 \otimes(r q) *)=\left(\sum_{i=1}^{n} e_{i} *\right) \otimes(r q) *=(r q) * \mathrm{QED}$

Corollary $5 D(C Q)$ is a path algebra. 


\subsection{Dual Path Algebras}

We define the comultiplication $\operatorname{map} \Delta: D(K Q) \otimes D(K Q) \rightarrow D(K Q)$ as following

$$
\Delta\left(p^{*}\right)=\sum_{q r=p} r^{*} \otimes q^{*}
$$

and for a trivial path $e_{i}{ }^{*}$ we have $\Delta\left(e_{i}^{*}\right)=e_{i} * \otimes e_{i}{ }^{*}$. The counit map $\varepsilon$ : $D(K Q) \rightarrow K$ is defined by the formula

$$
\varepsilon\left(\alpha^{*}\right)=\left\{\begin{array}{c}
1, \text { if } \alpha \in Q_{0} \\
0, \text { if } \alpha \text { is a pathof length } \geq 1
\end{array}\right.
$$

Lemma 6 The comultiplication $\Delta$ and the counit $\varepsilon$ satisfy

$$
\begin{aligned}
& \text { 1. }(\text { id } \otimes \Delta) \Delta=(\Delta \otimes i d) \Delta \\
& \text { 2. }(\text { id } \otimes \mathcal{E})^{\Delta}=(\varepsilon \otimes i d) \Delta .
\end{aligned}
$$

Proof Let $p^{*} \in D(K Q)$.

$$
\begin{aligned}
& (i d \otimes \Delta) \Delta\left(p^{*}\right)=(i d \otimes \Delta)\left(\sum_{q r=p} r * \otimes p^{*}\right)=\sum_{q r=p} r^{*} \otimes\left(\sum_{u v=q} v^{*} \otimes u *\right)=\sum_{u v r=p} r * \otimes v * \otimes u * \\
& (\Delta \otimes i d) \Delta\left(p^{*}\right)=(\Delta \otimes i d)\left(\sum_{q r=p} r^{*} \otimes p^{*}\right)=\sum_{u q=p}\left(\sum_{v r=q} r^{*} \otimes v^{*}\right) \otimes u^{*}=\sum_{u v r=p} r * \otimes v * \otimes u^{*}
\end{aligned}
$$

Moreover

$$
\begin{aligned}
& (i d \otimes \varepsilon) \Delta\left(p^{*}\right)=(i d \otimes \varepsilon)\left(\sum_{q r=p} r^{*} \otimes q^{*}\right)=p^{*} \\
& (\varepsilon \otimes i d) \Delta\left(p^{*}\right)=(\varepsilon \otimes i d)\left(\sum_{q r=p} r^{*} \otimes q^{*}\right)=p^{*}
\end{aligned}
$$

QED

Corollary $7 D(K Q)$ is a path coalgebra. 


\subsection{Opposite Quivers}

For a quiver $Q$ with set of vertices $\{1,2, \ldots, n\}$ we denote by $Q^{o p}$ the quiver having the same set of vertices. For each arrow $\alpha: i \rightarrow j$ in $Q$ there is an arrow $\alpha^{o p}: j \rightarrow i$ in $Q^{o p}$. For a path $p=\alpha_{1} \alpha_{2} \ldots \alpha_{m}$ define the path $p^{o p}$ as $p=\alpha_{m}^{o p} \alpha_{m-l}^{o p} \ldots$ $\alpha_{1}^{o p}$.

We define a bilinear map $(-,-): C Q \times K Q^{o p} \rightarrow \mathrm{K}$ by $(v, w)=\delta_{v w}$ (the Kronecker's delta) for any two paths $v, w \in Q$. This bilinear map defined above is degenerate in the following sense:

1. if $(v, w)=O$ for all $v \in C Q$, then $w=0$.

2. if $(v, w)=0$ for all $w \in K Q^{o p}$, then $v=0$.

This means that there exist two injective linear maps $\sigma: C Q \rightarrow D\left(K Q^{o p}\right)$ and $\tau: K Q^{o p} \rightarrow D(C Q)$ defined by $\sigma(v)(w)=(v, w)$ and $\tau(w)(v)=(v, w)$, for all $v \in$ $C Q$ and $w \in K Q^{o p}$.

Lemma 8 If $Q$ is any quiver, then

1. the injective morphism $K Q^{o p} \rightarrow D(C Q)$ is a morphism of algebras,

2. the injective morphism $\mathrm{CQ} \rightarrow \mathrm{D}\left(\mathrm{KQ}^{\mathrm{op}}\right)$ is a morphism of coalgebras.

Proof These are consequences of Corollary 5 and Corollary 7 QED

\section{$4 \quad$ Quivers with Relations}

Let $v \in C Q$, the orthogonal subspace to $v$ is the set $v^{\perp}=\{f \in D(C Q): f(v)=0\}$. More generally, for any subset $S \subseteq Q$, we define the orthogonal subspace to $S$ to be the space

$$
S^{\perp}=\{f \in D(C Q): f(S)=0\} .
$$

Since $K Q^{o p}$ can be embedded in $D(C Q)$ then we may consider the orthogonal subspace to $S$ in $K Q^{o p}$

$$
S^{\perp K Q^{o p}}=S^{\perp} \cap K Q^{o p}=\left\{w \in K Q^{o p}:(S, w)=0\right\} .
$$

Reciprocally, for any subset $T \subseteq D(C Q)$ the orthogonal subspace to $T$ in $C Q$, is defined by the formula 


$$
T^{\perp C Q}=\{v \in C Q: f(v)=0 \text { for all } f \in T\} .
$$

And if $T \subseteq K Q^{o p}$, then we write

$$
T^{\perp C Q}=\{v \in C Q:(v, w)=0 \text { for all } w \in T\} .
$$

For simplicity we write $\perp$ instead of $\perp C Q$.

Definition 9 Let $\left(Q^{o p}, I\right)$ be a quiver with relations. The bound path coalgebra of $\left(Q^{o p}, I\right)$ is defined by the subspace of $C Q$,

$$
C(Q, I)=\{a \in C Q:(a, I)=0\} .
$$

Lemma 10 Let $Q$ be a quiver and $C$ a relation subcoalgebra of $C Q$. Then $C^{\perp K Q^{o p}}$ is an admissible ideal of $K Q$.

Proof See [9, Corollary 4.3].

Proposition 11 Let $Q^{o p}$ be a quiver and $I$ an admissible ideal of $K Q^{o p}$, then $C(Q, I)=I^{\perp}$ is a relation subcoalgebra of $C Q$.

Proof See [9].

Lemma 12 Let $Q$ be a finite quiver and $C$ a finite dimensional relation subcoalgebra of $C Q$, then there exists an admissible ideal $I$ of $K Q^{o p}$ such that $C$ $=C(Q, I)$.

Proof Choose $I=C^{\perp K Q^{o p}}$. By [10, Theorem 2.2.1], $C=\left(C^{\perp K Q^{o p}}\right)^{\perp}=I^{\perp}$. And the later is $\mathrm{C}(\mathrm{Q}, \mathrm{I})$ by Proposition 11 . QED

Now we will state the main theorem which is is a generalization of the results in [6].

Theorem 13 (Main Theorem) Let $Q$ be a finite quiver.

1. If $I$ is an admissible ideal of $K Q^{o p}$ then $D\left(K Q^{o p} / I\right)$ $\cong I^{\perp}=C(Q, I)$.

2. If $C$ is a finite dimensional relation subcoalgebra of $C Q$ then $D(C) \cong K Q^{o p} / C^{\perp K Q^{o p}}$. 
Proof

1. Let $f \in D\left(K Q^{o p} / I\right)$. Then it is clear $f \in D\left(K Q^{o p}\right)$ such that $(f, I)=$ $O$, hence $f \in I^{\perp}$. Conversely, if $f \in I^{\perp}$, then we can identify $f$ with $f^{\prime} \in D\left(K Q^{o p} / I\right)$, where $f^{\prime}(\underline{p})=f(p)$ for all $\underline{p} \in K Q^{o p} / I$. This completes the proof.

2. By Lemma 12 and the statement above,

$$
C=C(Q, I)=I^{\perp} \cong D\left(K Q^{o p} / I\right) \text { where } I=C^{\perp K Q^{o p}} .
$$

Hence $D(C) \cong K Q^{o p} / I$.

\section{QED}

Corollary 14 Let $C$ be a relation subcoalgebra of $C Q$ and $D(C)$ be its dual. Then $D(C)$ is isomorphic to the bound path algebra of quiver $\left(Q^{o p}, I\right)$,i.e., the quiver of dual path subcoalgebra $D(C)$ can be obtained from the quiver $Q$ by reversing arrows.

Corollary 15 Let $K Q / I$ be a bound path algebra where $I$ is an admissible ideal of $K Q$, and $D(K Q / I)$ be its dual. Then $D(K Q / I)$ is isomorphic to the bound path coalgebra of quiver $\left(Q^{o p}, I\right)$ i.e., the quiver of dual bound path algebra $D(K Q / I)$ can be obtained from the quiver $Q$ by reversing arrows.

We give here some examples:

Example 16 Let $Q$ be the quiver

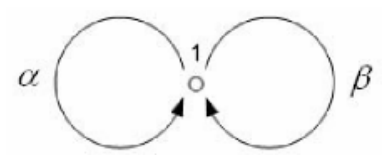

The ideal $I$ generated by $\alpha \beta-\beta \alpha, \beta^{2}, \alpha^{2}$ is an admissible ideal. The bound path algebra $K Q / I$ is 4-dimensional with basis $\left\{\overline{e_{1}}, \bar{\alpha}, \bar{\beta}, \overline{\alpha \beta}\right\}$. The dual of $K Q / I$ is $C\left(Q^{o p}, I\right)$, the relation subcoalgebra of $C Q^{o p}$ where $Q^{o p}$ is the same quiver as $\mathrm{Q}$.

Example 17 Let $Q$ be the quiver 
Quivers of Bound Path Algebras and Bound Path Coalgebras 161

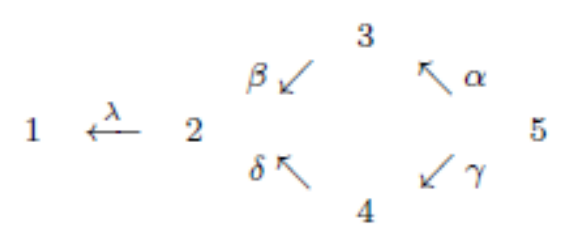

bound by the relation $\alpha \beta=\gamma \delta$. Then the dual of $K Q / I$ is the relation subcoalgebra $C\left(Q^{o p}, I\right)$ where $Q^{o p}$ is the quiver

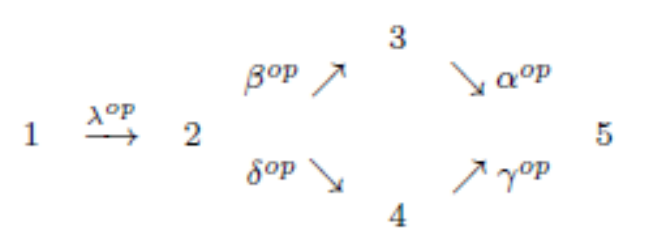

Example 18 Let $Q$ be the quiver

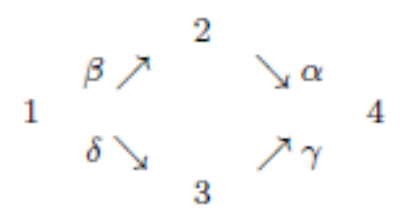

and $C$ be the relation subcoalgebra generated by $\{\beta \alpha+\gamma \delta\}$. Then $D(C)$, the dual of $C$ is $K Q^{o p} / C^{\perp K Q^{o p}}$ where $Q^{o p}$ is the following quiver:

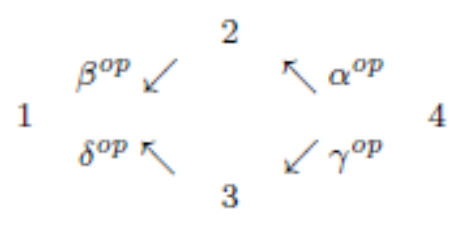

As conclusion, from the relation between path coalgebras and path algebras, we can study more on coalgebras and algebras using their quivers. We may also conclude that dual of basic algebras are pointed coalgebras and dual of pointed coalgebras are basic algebras. 


\section{Acknowledgement}

We would like to thank Pudji Astuti for various discussion and suggestions. The authors are supported by Hibah Penelitian Fundamental DIKTI based on Surat Perjanjian Pelaksanaan Riset No. 0051X/K01.20/SPKLPPM/I/2009.

\section{References}

[1] Assem, I., Simson, D. \& Si Skowronski, A., Elements of the Representation Theory of Associative Algebras 1, London Mathematical Society Student Texts 65, Cambridge University Press, 2006.

[2] Muchtadi-Alamsyah, I. Algebras and quivers, Proceeding ISSM Paris, 2005.

[3] Chin, W., A brief introduction to coalgebra representation theory, in Hopf Algebra, Lecture Notes in Pure and Appl.Math, 237, pp.109-131, Marcel Dekker, New York, 2004.

[4] Auslander, M., Reiten, I. \& Smaloe, S.O., Representation Theory of Artin Algebras, Cambridge Univ Press, 1995.

[5] Chin, W., Si Montgomery, S., Basic Coalgebras, Modular interfaces (Reverside, CA, 1995), 41-47, AMS/IP Stud. Adv. Math. 4, Providence, RI:Amer.Math.Soc., 1997.

[6] Muchtadi-Alamsyah, I. \& Garminia,H. Quivers of Path Algebras and Path Coalgebras, Proceeding IndoMS International Conference on Mathematics and Its Applications, 2009.

[7] Woodcock, D. Some categorical remarks on the representation theory of coalgebras, Communication in Algebra, 25, pp. 2775-2794, 1997.

[8] Simson, D. Path coalgebras of quivers with relations and a tame-wild dichotomy problem for coalgebras, Lecture Notes in Pure and Applied Mathematics, 236, pp. 465-492, 2005.

[9] Jara, P., Merino, L.M. \& Navarro, G. On Path Coalgebras of Quivers with Relations, Colloq. Math., 102, pp. 49-65, 2005.

[10] Abe, E., Hopf Algebras, Cambridge University Press 1977. 